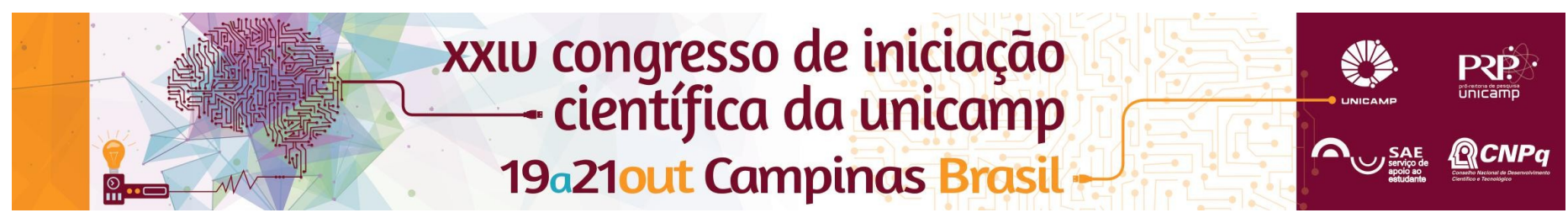

\title{
Analysis and Simulation of Computacional Mechanisms for Action Selection
}

\author{
Ricardo R. Gudwin, Bárbara R. Rosado*
}

\begin{abstract}
Action selection is one of the basic problems studied in Artificial Intelligent Systems. Many of the solutions involve animal inspiration. An animal in its habitat takes several decisions all the time, using as a basis their sensory perceptions, motivations and instincts. Artificial mechanisms of action selection allow artificial creatures, such as robots or characters in computer games, to make these decisions autonomously. This project aims at analyzing and studying Computational Mechanisms for Action Selection used in cognitive architectures. We focused mainly on the algorithms described in the doctoral thesis of Toby Tyrrell $\left(199^{3}\right) 1$. The work of Tyrrell analyzes four different action selection mechanisms: the mechanisms based on drives, the Maes algorithms, Lorenz and Rosenblatt \& Payton. In this work, we studied the strategies mentioned by Tyrrell $\left(199^{3}\right) 1$ in order to reproduce two of these mechanisms, the mechanisms based on drives and Maes algorithm, and thus compare the results with those obtained by him. The algorithms were encoded using the Java language and made available as a part of the CST - Cognitive Systems Toolkit, a software toolkit being developed by Prof. Gudwin's research team at FEEC/UNICAMP.
\end{abstract}

\section{Key words:}

Cognitive Architecture, Artificial Action Selection Mechanisms, Control of Intelligent Autonomous Agents.

\section{Introduction}

Cognitive architectures are computational tools inspired by cognitive models, which serve for the control of intelligent autonomous agents. These computational architectures are able to provide basic infrastructure for the construction of "artificial minds" to agents, both physical (robots and autonomous vehicles), and virtual ("players" in computer games). These architectures are used for cognitive models of the human mind in order to implement computational versions of skills or cognitive functions in artificial creatures. Action Selection is one of the topics covered by a cognitive architecture, among other methods based on cognitive models for implementation of algorithms for artificial minds. In this work, instead of directly reproducing the experiments conducted by Tyrrell, we used a virtual environment developed by Professor Ricardo Gudwin's research group at FEEC-UNICAMP, called WorldServer3D ${ }^{2}$. In this virtual environment, developed using the Java programming language, you can create virtual creatures, controlled by their sensors and actuators, and accessible via Sockets. The mechanisms studied by Tyrrell were used to control these creatures within the virtual environment through a Proxy Server to the 3D World. The WorldServer3D Proxy Library is a support that makes all the connection to the World Server 3D, and send and receive commands in high-level Java objects.

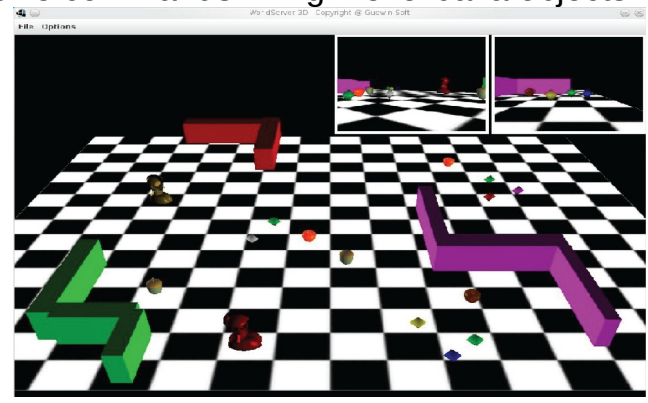

Image 1: WorldServer 3D environment structure. Results and Discussion

Image 2 illustrates our version of the Drive Mechanism applied in the control of the virtual creature, in terms of codelets and memory objects (the basic functional types in the CST toolkit). The "drives" are the internal needs of a creature and are considered as the motivation of behavior. In this diagram the drives are represented by memory objects like hungerMO and ambitionMO. The intensity of a drive measures how much that inner need is important for the agent. The image represents just one behavior of the proposed mechanism.

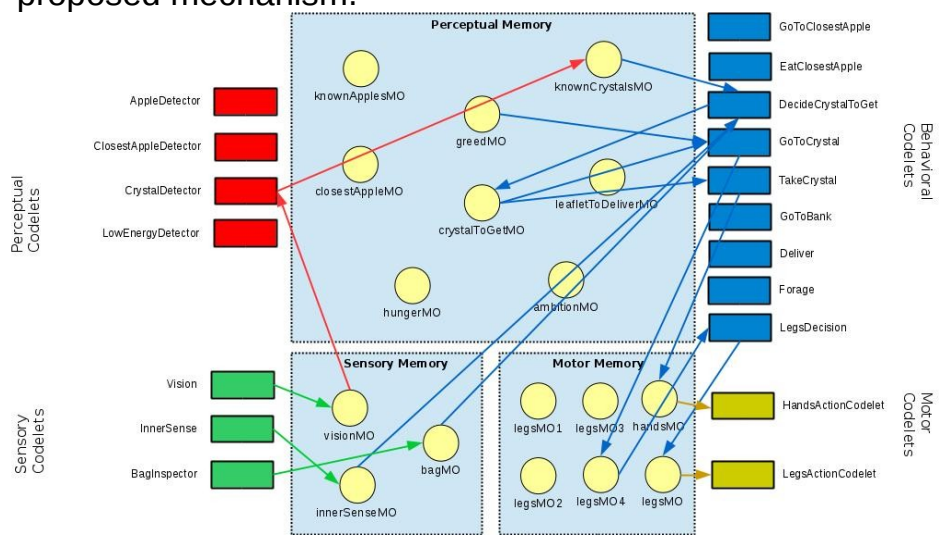

Image 2: Codelets and Memory Objects structure of Drives Mechanism.

\section{Conclusions}

This work presents the implementation and test of two different action selection mechanisms which were developed as a part of CST, the Java-based toolkit for the construction of cognitive architectures, which is being developed by the research group of Professor Ricardo Gudwin. The code made available on GitHub might assist other researchers interested on the study of action selection mechanisms in artificial creatures.

\section{Acknowledgement}

This work was supported by PIBIC. Quota 2015.

\footnotetext{
1 Tyrrell, T. - Computational Mechanisms for Action Selection. Doctoral Thesis, University of Edinburgh, 1993.

${ }^{2}$ Castro, E. C. .et.al. A Scene-Based Episodic Memory System for a Simulated Autonomous Creature. International Journal of Synthetic Emotions, 4(1), 32-64, January-June 2013.
} 